\title{
Editorial
}

\section{Special issue dedicated to Daizhan Cheng}

Dr. Daizhan Cheng is a full professor of the Key Lab of Systems and Control, Academy of Mathematics and Systems Science, Chinese Academy of Sciences, and was the former Editor-in-Chief of this journal, Control Theory and Technology (whose original title was Journal of Control Theory and Applications before 2013) during 2003-2013. He was born in March 1946 and got his Ph.D. degree from Washington University, USA. Prof. Cheng contributed much to several control fields, especially to nonlinear control systems, networked or switched systems, logic dynamic systems, and numerical realization of control design, and published over 10 books and 120 journal papers. Because of his outstanding research achievement, he won National Natural Science Award of China twice (in 2008 and 2014, respectively) and Automatica 2008-2010 Theory/Methodology Best Paper Award (in 2011). He is also an IEEE Fellow (2005-) and an IFAC Fellow (2008-) for his international influence.

This special issue is to honor the 70th birthday of Prof. Daizhan Cheng, our former editor-in-chief. It includes wide variety of papers fully corresponding with the extent of Prof. Cheng-s professional interests. In fact, the papers mainly cover many new and challenging research areas in systems and control, which can be roughly divided into two topics: network-based control and control of nonlinear or delayed systems. For the first topic, there are 5 papers. The first article gives an overall picture about cyber-physical system security, while the second one studies distributed non-cooperative MPC. Next, two papers on multi-agent consensus follow, with one on observer-based tracking control and the other on communication delays. Then the fifth paper investigates the networked epidemic dynamics using matrix expression. For the second topic, 4 papers are included. The first one discusses the output regulation for delayed systems, while the second one handles the stabilization of stochastic nonlinear systems. Then the third paper presents an effective robot control with human-robot interaction, and finally, the fourth paper shows an exciting path planning approach for drones.

There is no other way to conclude this editorial than to express our gratitude. First, we want to thank all the contributors whose papers appear in this special issue. Then we want to thank the editorial office who gave us the precious opportunity and also provided great help for this issue. Above all, we want to thank Prof. Cheng for his distinguished contribution to this journal and, more important, for the privilege that we can spend a long and fruitful time with him as his former PhD students and as his current colleagues.

Happy birthday to Prof. Cheng!

\section{Guest editors:}

Yiguang Hong, Zairong Xi

Academy of Mathematics and Systems Science

Chinese Academy of Sciences

(c) 2016 South China University of Technology, Academy of Mathematics and Systems Science, CAS, and Springer-Verlag Berlin Heidelberg 